\title{
DIRETRIZES BRASILEIRAS PARA O MANEJO DA AGITACÃO PSICOMOTORA: CUIDADOS GERAIS E AVALIAĈ̣̃O*
}

\section{BRAZILIAN GUIDELINES FOR THE MANAGEMENT OF PSYCHOMOTOR AGITATION: GENERAL CARE AND ASSESSMENT}

\section{Resumo}

Neste primeiro artigo, apresentamos os aspectos gerais dessa diretriz. Na sequência, apresentamos os cuidados gerais do ambiente e equipe e, posteriormente, a avaliação de pacientes agitados, principalmente focada no diagnóstico diferencial.

Número de registro da revisão sistemática: CRD42017054440.

Palavras-chave: Agitação psicomotora, agressividade, tranquilização farmacológica, emergência psiquiátricas, avaliação de risco, manejo não farmacológico.

\section{Abstract}

In this first article, we address the general aspects of these guidelines. Subsequently, we describe the characteristics of general care, related to both the setting/facilities and the team. Finally, the assessment of agitated/aggressive patients is discussed, with a focus on differential diagnosis.

Systematic review registration: CRD42017054440.

Keywords: Psychomotor agitation, aggression, pharmacological tranquilization, psychiatric emergencies, risk assessment, non-pharmacological management.

\footnotetext{
* Texto adaptado do artigo originalmente publicado em inglês no Brazilian Journal of Psychiatry, com a seguinte referência: Baldaçara L, Ismael F, Leite V, Pereira LA, Dos Santos RM, Gomes Júnior VP, et al. Brazilian guidelines for the management of psychomotor agitation. Part 1. Non-pharmacological approach. Braz J Psychiatry. 2019;41:153-67. http://dx.doi.org/10.1590/1516-4446-2018-0163
}

\section{INTRODUÇ̃̃o}

A emergência é qualquer situação em que haja ameaça à vida e requeira intervenção imediata. A emergência psiquiátrica é qualquer mudança no comportamento do paciente, existindo um risco significativo para si ou para outros, necessitando de uma intervenção terapêutica imediata (em questão de minutos ou algumas horas) para evitar danos. Entre as emergências mais prevalentes, estão: comportamento suicida, episódios depressivos ou maníacos, automutilação, julgamento gravemente prejudicado, autonegligência grave, intoxicação ou abstinência, agitação psicomotora e agressividade ${ }^{1-4}$.

A agitação psicomotora é um fenômeno frequente e uma condição clinicamente relevante em pacientes com transtornos psiquiátricos, não apenas em situações de urgência, mas também em ambientes hospitalares e ambulatoriais $^{5}$. A agitação com ou sem agressividade é responsável por 2,6 a 52\% de todas as emergências psiquiátricas em todo o mundo ${ }^{6-12}$ e 23,6 a 23,9\% ( 24\%) das emergências psiquiátricas no Brasil ${ }^{13,14}$.

Diversas diretrizes sobre o manejo da agitação psicomotora foram produzidas, como, por exemplo: Best Practices in Evaluation and Treatment of Agitation, ou Projeto BETA, desenvolvido pela American Association for Emergency Psychiatry ${ }^{15}$; Critical Issues on the Diagnosis and Management of the Adult Psychiatric Patient in the Emergency Department, da American College of Emergency Physicians (ACEP) ${ }^{16}$; Violence - The Short-Term Management of Disturbed/Violent Behavior in Psychiatric In-Patient Settings and Emergency 
Departments, uma diretriz de 2005 desenvolvida pelo National Institute for Clinical Excellence (NICE) ${ }^{17}$; e o Assessment and Handling of Agitation in Psychiatry consensus statement ${ }^{5}$.

No Brasil, apesar do artigo de 2010 de Mantovani et al. ${ }^{18}$, que trata sobre o manejo de pacientes violentos ou agitados, até o momento não há diretrizes atualizadas baseadas em evidências que se apliquem à realidade desse país. $\bigcirc$ objetivo deste estudo foi desenvolver e apresentar recomendações essenciais para o manejo não farmacológico de pacientes com agitação psicomotora no Brasil.

\section{ESTRUTURA DA EQUIPE (CONDIÇÕES AMBIENTAIS E DA EQUIPE)}

É importante ressaltar que o ambiente ideal para o atendimento em crise nem sempre estará disponível, já que as emergências podem ocorrer em qualquer lugar, sem aviso prévio. Nesse caso, a equipe deve utilizar os recursos disponíveis e seguir as recomendações gerais presentes nessa diretriz, encaminhando o paciente para um serviço de emergência o mais rápido possível. A preocupação inicial deve ser tanto com a segurança do paciente quanto das pessoas ao seu redor ${ }^{5,19}$. Os médicos e outros membros da equipe nunca devem se colocar em risco (por exemplo, permanecer em uma sala fechada sem saída fácil) (D) 5 .

O ambiente físico é importante para o manejo do paciente agitado. Uma área específica deve ser reservada para lidar com pacientes psiquiátricos. Esse local deve conter uma equipe de enfermagem treinada, com consultórios e banheiros bem ventilados $(D)^{20,21} \mathrm{e}$ iluminados, com objetos de orientação adequados, tais como relógios e calendários para pacientes que estejam confusos $(D)^{20}$. As instalações precisam de controle climático adequado da temperatura, da luz, da ventilação e do ruído $(D)^{21}$.

Os móveis devem estar dispostos na sala de espera e no consultório, de modo a proporcionar acesso fácil às saídas, tanto dos pacientes quanto da equipe (D). A possibilidade de remover os móveis rapidamente permite que um ambiente seguro seja estabelecido com rapidez (D)22. No entanto, alguns serviços de emergência preferem móveis fixados ao chão ou à parede, para que assim os pacientes não possam utilizá-los como armas, o que, infelizmente, pode trazer uma falsa sensação de segurança (D) ${ }^{22}$.
As rotas de passagem devem ser acessíveis e devese evitar os excessos de estímulos sensoriais, tais como sons altos, cores vivas e calor ou frio extremos (D) ${ }^{20,22}$. Os pacientes precisam sentir-se confortáveis com o mínimo possível de estímulos externos, em quartos silenciosos e individuais e com o menor tempo de espera possível $(D)^{5,20,21,23}$. A sala reservada para urgências psiquiátricas precisa de uma mesa e de cadeiras para o profissional, para o paciente e para os seus familiares, de uma maca e de uma pia para lavar as mãos ${ }^{4,20,24}$.

Deve haver uma saída atrás do profissional que atende - paciente, que deve estar desobstruída caso haja alguma ameaça que não possa ser manejada e haja a necessidade de fuga (D)4. É preciso lembrar que médicos e funcionários lidam com pacientes que podem estar em crise e comportar-se de forma imprevisível (D) ${ }^{4}$. Quaisquer itens ou objetos potencialmente perigosos, que possam ser utilizados como arma, tais como canetas, objetos pontiagudos ou luminárias de mesa, devem ser removidos ou guardados de forma segura (D) 5 . Os médicos devem manter-se próximos de qualquer outro objeto que não possa ser removido (D)².

Frequentemente, a agitação tem uma causa orgânica, e esses pacientes podem evoluir com complicações inerentes à sua doença ou até mesmo ao próprio tratamento. $\bigcirc$ equipamento de emergência deve estar facilmente disponível (oxigênio, ressuscitadores manuais, materiais para intubação, aspirador de secreção, nebulizador, carrinho ou bandeja com desfibrilador) $(D)^{20}$. O local também deve estar equipado para realizar exames laboratoriais padrões e adicionais, tais como glicemia capilar, gasometria e eletrocardiograma (D)5.

Também devem estar disponíveis materiais para a contenção física de punho, tornozelo e tórax $(D)^{2}$. As áreas de observação devem estar equipadas com camas com cabeceira elevada e barras fixas para a fixação das contenções, se necessário. É desaconselhável manter os pacientes sob observação em macas $(D)^{20}$. A organização do local deve ser feita de forma a facilitar a observação, com campo de visão desobstruído, sem pontos cegos $(\mathrm{D})^{21}$.

A ocupação não deve exceder o número de leitos disponíveis, pois a tensão entre os pacientes e a equipe tende a aumentar quando há esse excesso (D) ${ }^{21}$. Sempre que a equipe estiver envolvida no manejo de pacientes 
violentos, todos os esforços devem ser feitos para manejar da forma menos restritiva o ambiente (D) ${ }^{21}$. Um modelo frequentemente utilizado é a unidade de terapia intensiva psiquiátrica (UTIP) ${ }^{21,25}$. Trata-se de uma pequena unidade de observação especializada, em que há equipe treinada, espaço, equipamento e segurança adequados $^{21,25}$. Foram observados resultados mais favoráveis em pacientes tratados em uma UTIP, quando comparados aos de uma unidade psiquiátrica aguda (B).

\section{Características da EQUIPE RESPONSÁVEl PELOS PACIENTES COM AGITAÇÃO PSICOMOTORA}

A equipe deve ser treinada e basear-se em protocolos terapêuticos que descrevam todas as etapas do atendimento ao paciente, incluindo o papel de cada profissional na resolução do problema (D) ${ }^{20}$.

O manejo adequado dos casos agudos envolve a capacidade de realizar várias tarefas e tolerar mudanças rápidas nas prioridades dos pacientes ${ }^{22}$. Os médicos que atuam com pacientes agitados devem avaliar se estão aptos para esse trabalho. Pacientes agitados podem desafiar a autoridade, a competência e até mesmo as credenciais do clínico e podem ser muito bons em detectar e explorar as vulnerabilidades do médico ${ }^{22}$. Os profissionais que trabalham com pacientes agitados devem reconhecer e controlar problemas de contratransferência ${ }^{22}$. A menos que o médico e a equipe entendam as suas próprias vulnerabilidades, comportamentos como retaliar, argumentar ou ficar na defensiva só servirão para piorar ainda mais a situação. Os clínicos também precisam reconhecer seus limites, aprendendo a procurar ajuda adicional, se necessário (D)22.

Recomendamos o uso de jaleco, roupas neutras e não provocativas e crachás de identificação. Os profissionais devem evitar adereços longos, como brincos e colares, e cabelos soltos. O motivo para tais recomendações é desencorajar ataques de pacientes mais agressivos $(D)^{26}$. Deve ser mantida uma distância adequada dos pacientes agitados, para protegê-los e também à equipe. O contato visual direto e prolongado e os movimentos bruscos podem ser vistos como uma ameaça e devem ser evitados $(D)^{26}$. É essencial que o paciente se sinta sempre respeitado. Deve-se ponderar criteriosamente o papel do ouvinte empático e o da figura de autoridade, demonstrar conhecimento sobre o assunto e transmitir confiança e segurança aos pacientes e familiares (D) ${ }^{26}$. Da mesma forma, comportamentos provocativos e julgadores devem ser evitados (D) ${ }^{26}$.

\section{AvaLIAÇõES}

A chegada de um paciente agitado a um departamento de emergência requer uma avaliação rápida e eficaz da situação para implementar, o mais rápido possível, o melhor curso de ação. Os principais objetivos no cuidado de um paciente com agitação psicomotora são: triagem e avaliação da gravidade; anamnese objetiva e subjetiva; exame físico e neurológico; exame psiquiátrico; diagnóstico diferencial; tranquilização rápida; encaminhamento e orientação.

No caso de um paciente agitado que apresente comportamento violento, pode ser difícil executar todas essas etapas assim que o paciente chegar ao serviço. Frequentemente, o paciente precisará ser tranquilizado antes que uma avaliação possa ser feita.

Como o tempo é importante, em uma emergência, o uso de quatro perguntas básicas agilizam o primeiro atendimento (D) ${ }^{4}$, conforme descrito abaixo e na Figura 1.

A primeira pergunta que se deve realizar é: o que está acontecendo? É necessário listar as mudanças no comportamento que são motivo de preocupação. Às vezes, os pacientes e seus familiares podem ser prolixos ou fornecer informações que não são verdadeiras. É importante determinar as alterações agudas no comportamento que podem colocar o paciente ou terceiros em risco. Também é necessário eliminar quaisquer causas orgânicas que possam se apresentar como alterações psiquiátricas ou comportamentais ${ }^{26,27}$.

A segunda pergunta que se deve fazer é: há quanto tempo? É preciso descobrir se o paciente está exibindo esses comportamentos diferentes por período curto ou longo e se, de fato, houve alguma mudança importante e aguda no comportamento. Lembre-se de que mesmo no caso de pacientes com uma longa história de agitação, a situação pode ser urgente. Mudanças repentinas de comportamento também podem ser causadas por fatores orgânicos²6.

A terceira pergunta seria: por que hoje? É importante descobrir o motivo pelo qual escolheram esse momento para procurar ajuda de emergência. O quarto questionamento a ser realizado é: quais são as possíveis 
hipóteses diagnósticas ou os diagnósticos temporários? É preciso listar as hipóteses diagnósticas para iniciar o processo de diagnóstico diferencial e identificar a melhor conduta. Na sala de emergência, use preferencialmente diagnósticos sindrômicos, como transtorno psicótico ou transtorno do humor, pois a velocidade não permitirá um diagnóstico mais detalhado. Preste atenção às possíveis causas de agitação ${ }^{26}$.

\section{TRIAGEM E AVALIAÇÃO DA GRAVIDADE}

A triagem começa no primeiro contato com o paciente agitado e envolve observação direta e uma tentativa de estabelecer um diálogo $(D)^{28}$. O objetivo é distinguir os pacientes que requerem atenção imediata daqueles que podem esperar a sua vez ou ser encaminhados para um atendimento ambulatorial.

No Projeto BETA, de Nordstrom et al. ${ }^{29}$, é sugerido o uso das escalas de avaliação ancoradas em comportamentos
(BARS) para a triagem e a tomada de decisão em ambientes não médicos. Basicamente, pacientes com níveis reduzidos de consciência, que não respondem ou não podem ser despertados, devem ser encaminhados imediatamente para um departamento de emergência médica.

Pacientes sonolentos que respondem normalmente ao contato verbal ou físico, pacientes sonolentos que parecem sedados ou pacientes violentos que necessitam de contenção física devem ser enviados para uma unidade de emergência médica ou para um serviço de emergência psiquiátrica com apoio de ambulância (D). Para pacientes que apresentam sinais de agitação que não exijam contenções físicas ou que se acalmem quando instruídos, deve-se tentar abordagem verbal para reduzir a agitação (técnica de desescalada verbal). Se isso falhar, o paciente deve ser encaminhado ao serviço de emergência (D) ${ }^{29}$.

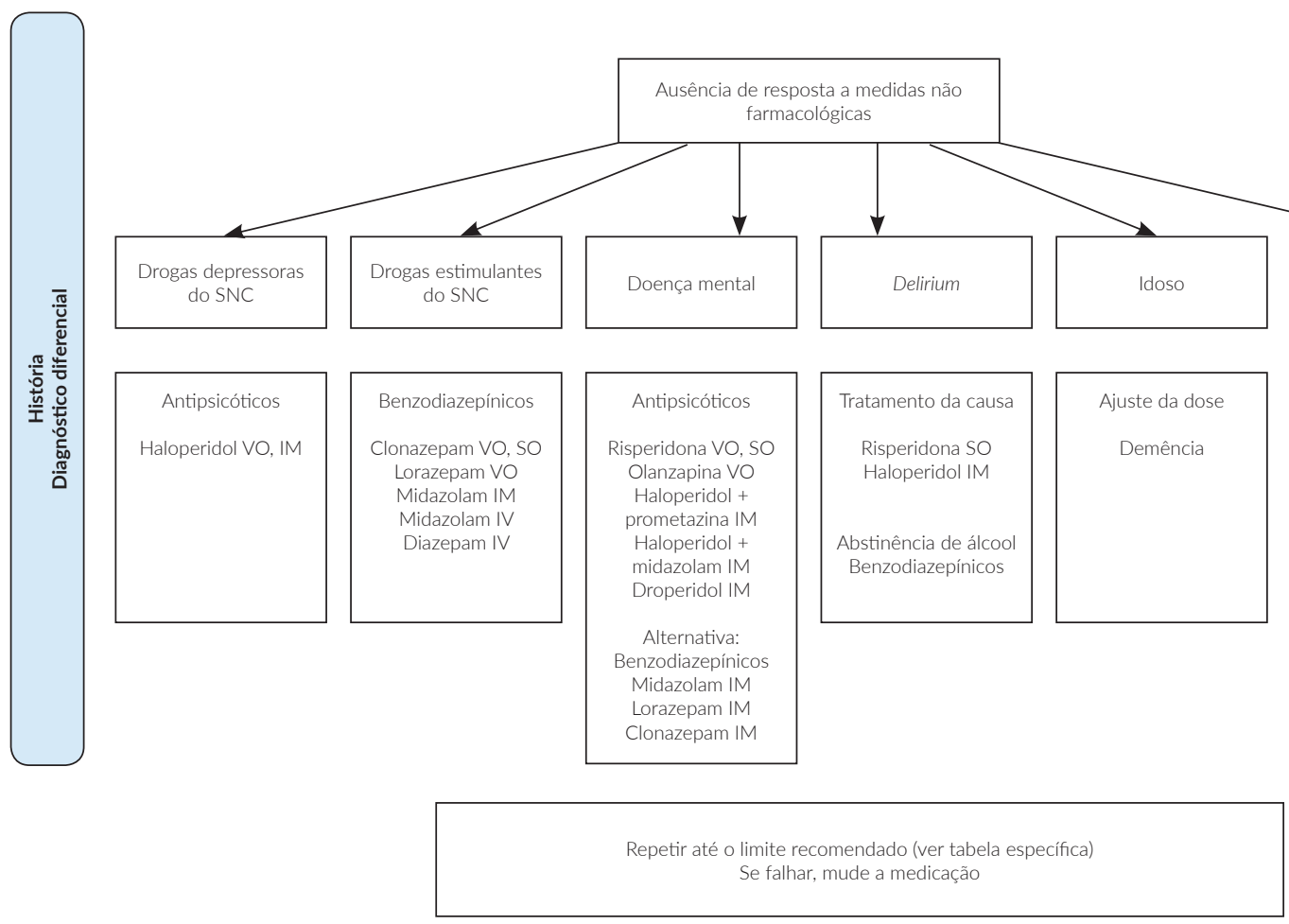

Figura 1 - Processo de seleção do estudo. SNC = sistema nervoso central; $\vee O$ = via oral; SO = solução oral; $\mid \mathrm{M}=$ intramuscular. 
Somente os pacientes que se acalmem e/ou respondam às instruções verbais devem ser mantidos nas salas de espera. Todos os outros devem ser tratados imediatamente nos serviços de emergência (D). Em algumas situações, emergenciais ou não (D) ${ }^{29}$, a agitação pode ser considerada mais grave e podem indicar alterações comportamentais secundárias a doenças físicas. Essas situações estão listadas no Quadro 1.

Pacientes que chegam a uma unidade de emergência devem ser avaliados por um psiquiatra ou enfermeiro para estabelecer a vontade e a capacidade do paciente de dialogar e informar dados sobre o comportamento e os seus tratamentos anteriores (D) ${ }^{30}$. Isso começa com uma entrevista e um exame psiquiátrico. Os procedimentos iniciais oferecem uma oportunidade para a investigação clínica, com foco nos sintomas menos visíveis, como sintomas negativos ou cognitivos, e com a investigação de quaisquer sinais de doença orgânica (D) ${ }^{30}$. No entanto, como a agitação psicomotora atrai mais atenção, pode-se realizar uma

Quadro 1 - Fatores que sugerem distúrbios orgânicos subjacentes em pacientes agitados 21,29

\begin{tabular}{l}
\hline Sintomas agudos em pacientes com 45 anos ou mais \\
Sinais vitais alterados \\
Déficits neurológicos focais \\
Evidência de trauma na cabeça \\
Intoxicação ou abstinência de substâncias \\
Doença física preexistente \\
Rebaixamento do nível de consciência \\
Perda de memória \\
Alucinações visuais \\
Tentativas de suicídio \\
Sudorese, tremor, palidez \\
Cefaleia severa \\
Rigidez muscular ou fraqueza extrema \\
Intolerância ao calor \\
Perda involuntária de peso \\
Dificuldade em respirar \\
Convulsões \\
Psicose de início recente
\end{tabular}

avaliação clínica ${ }^{30}$. A entrevista inicial deve ser seguida de um exame físico e mental para determinar a melhor conduta.

A triagem inicial deve incluir: avaliação da capacidade do paciente de colaborar, com base na observação e interação verbal (espera e diálogo); em um ambiente tranquilo, deve-se realizar uma avaliação da urgência e da necessidade de encaminhamento imediato a um serviço de emergência médica ou psiquiátrica; na sala de espera, é preciso fazer uma avaliação da capacidade do paciente em esperar e/ou nível de prioridade; finalmente, a equipe deve reunir dados preliminares para iniciar imediatamente a conduta no consultório médico ou na sala de emergência. Avaliações de risco padronizadas ajudarão a garantir a segurança do ambiente clínico ${ }^{21}$.

1) Avaliação da gravidade. Toda a abordagem desde a triagem até a avaliação inicial, os métodos de tranquilização, a evolução subsequente, o diagnóstico diferencial e os critérios para alta e encaminhamento - deve ser registrada no prontuário do paciente (D). Ao cuidar de um paciente agitado, sinais vitais e exame físico, mesmo que simplificados, devem ser realizados periodicamente (D).

As escalas permitem avaliações objetivas e padronização dentro da equipe; além disso, tornam possível monitorar efetivamente a abordagem. A Tabela 1, a seguir, lista as principais escalas que podem ser usadas nessas situações.

Essas escalas foram selecionadas porque possuem poucos campos e podem ser aplicadas em qualquer local. Todas elas foram utilizadas anteriormente para a avaliação de agitação de pacientes. Escalas que apenas avaliam a sedação não são incluídas, nem escalas longas e autoadministradas (é improvável que um paciente agitado possa responder a um questionário). Para escalas para avaliar agitação e violência no comportamento, consulte a Tabela 1. Na presente diretriz, as escalas selecionadas foram originalmente desenvolvidas em inglês, podendo ser usadas por profissionais de saúde fluentes nesse idioma, além de outras escalas validadas para o português do Brasil. 
2) Risco de o paciente tornar-se agressivo. A agitação é uma situação dinâmica, que pode rapidamente se transformar em agressão ou em um comportamento violento ${ }^{5,49}$. A melhor conduta para o gerenciamento do comportamento agressivo ou violento é aquele que se concentra na identificação precoce de indivíduos em risco de escalada de agitação. Embora a literatura sugira que a maioria dos comportamentos violentos surja sem aviso,50,51, alguns autores sugerem que episódios agressivos podem ser associados a fatores de risco específicos e são precedidos por sinais de alerta comportamentais ${ }^{5}$, listados na Tabela 2. Várias ferramentas de avaliação foram projetadas para avaliar o risco de agressão/ violência, conforme mostrado na Tabela 3.

Fatores considerados protetores incluem harmonia entre o pessoal (bom ambiente de trabalho) e a presença de enfermeiros (sexo masculino) (3A) ${ }^{53}$. Aqui gostaríamos de salientar a importância de uma equipe treinada e coesa, com membros que se respeitam e seguem protocolos.

\section{A HISTÓRIA DO PACIENTE (ANAMNESE OBJETIVA E SUBJETIVA)}

$O$ foco deve ser o paciente, que precisa ser ouvido. A razão para procurar ajuda médica pode ser diferente para o paciente e seu acompanhante. Muitas vezes, familiares ou acompanhantes tentam intervir ou até mesmo irritam o paciente de propósito. Tais práticas devem ser desencorajadas. Sempre que possível, os pacientes devem ser avaliados em um ambiente silencioso e seguro. Pode ser aceitável para a família que acompanhantes entrem na sala de exames antes do paciente para relatar o caso, mas isso pode não ser produtivo (D). Se os membros da família insistirem em permanecer na sala de exame, devem ser instruídos a não interferirem. Em algumas situações, os familiares devem ser solicitados a deixar a sala, para permitir que a conversa flua (D). O tempo disponível para isso e as condições no departamento de emergência psiquiátrica nem sempre podem ser as melhores. Por essa razão, o histórico do paciente deve se concentrar nas quatro questões propostas acima (D).

As seguintes informações devem ser obtidas: queixa principal, história da doença atual, história da doença psiquiátrica, histórico médico, uso e abuso de substâncias, rede de apoio psicossocial, história familiar e avaliação do estado mental ${ }^{28}$.

\section{EXAME FísICO E NEUROLÓGICO (D)}

O exame físico é um componente essencial da avaliação de pacientes com agitação psicomotora. Contudo, a princípio, pode não ser possível realizar

Tabela 1 - Escalas para avaliar agitação e agressividade/estado violento

\begin{tabular}{|c|c|c|}
\hline Escala & Validada no Brasil & Nível de evidência \\
\hline Escala da gravidade da agitação (ASS) ${ }^{31}$ & Não & $2 \mathrm{~B}(\mathrm{~B})$ \\
\hline Escalas de avaliação ancoradas em comportamentos (BARS) ${ }^{32}$ & Não & $2 \mathrm{~B}(\mathrm{~B})$ \\
\hline Lista de verificação da violência de Brøset (BVC) ${ }^{33,34}$ & $\operatorname{Sim}^{35}$ & $2 \mathrm{~B}(\mathrm{~B})$ \\
\hline Escala de impressão clínica global para agressão (CGI-A) ${ }^{36}$ & Não & $2 \mathrm{~B}(\mathrm{~B})$ \\
\hline Gerenciamento do histórico clínico de riscos-20 (HCR-20) $)^{37}$ & $\operatorname{Sim}^{38}$ & $2 \mathrm{~B}(\mathrm{~B})$ \\
\hline Escala de agressão aberta $(\mathrm{OEA})^{39}$ & Não & $2 \mathrm{~B}(\mathrm{~B})$ \\
\hline Escala de gravidade da agitação (OASS) ${ }^{40}$ & Não & $2 \mathrm{~B}(\mathrm{~B})$ \\
\hline Componente de excitação da escala de síndrome positiva e negativa ${ }^{41}$ & Não & $2 \mathrm{~B}(\mathrm{~B})$ \\
\hline Escala de agitação-sedação de Richmond (RASS) ${ }^{42}$ & $\operatorname{Sim}^{43}$ & $2 \mathrm{~B}(\mathrm{~B})$ \\
\hline Escala de sedação e agitação (SAS) ${ }^{44}$ & $\operatorname{Sim}^{43}$ & $2 \mathrm{~B}(\mathrm{~B})$ \\
\hline Ferramenta de avaliação de sedação (SAT) ${ }^{45}$ & Não & $1 B(A)$ \\
\hline Escala de observação de agressão revista (SOAS-R) ${ }^{46,47}$ & Não & $2 \mathrm{~B}(\mathrm{~B})$ \\
\hline Lista de verificação de rastreamento de violência (VSC)48 & Não & $2 \mathrm{~B}(\mathrm{~B})$ \\
\hline
\end{tabular}




\section{ARTIGO DE REVISÃO}

Tabela 2 - Fatores de risco para agressividade/comportamento violento

\section{Fatores}

Nível de evidência

Fatores demográficos

Sexo masculino ${ }^{11,52}$, solteiro ou divorciado ${ }^{11,52}$, menor nível de escolaridade ${ }^{11}$, desemprego ${ }^{11}$

Ser jovem (o risco diminui com o aumento da idade) ${ }^{52,53}$

\section{História pregressa}

Atividades ilegais que resultam em encarceramento ${ }^{11}$, uso frequente de serviços de emergência ${ }^{11}$

Condições de admissão

Trazido pela polícia, ambulância, cuidador ou sob ordem judicial ${ }^{11}$

\section{Sinais e sintomas}

Ideação ou tentativa de suicídio ${ }^{11,53}$, mania ou hipomania ${ }^{11}$, tristeza ${ }^{11}$, psicose ${ }^{11,53,54}$, alucinações ${ }^{11}$, ideação bizarra ${ }^{11}$, ansiedade ${ }^{11}$

Excitação geral dos sistemas corporais (aumento das frequências cardíaca e respiratória, dilatação das pupilas $)^{54}$, processos de pensamento pouco $\operatorname{claros}^{54}$, falta de concentração $0^{54}$

\section{Diagnóstico}

Transtorno de adaptação ${ }^{11}$, transtorno de personalidade ${ }^{11}$, transtorno de humor ${ }^{11}$, transtorno psicótico $11,54,55$, uso/abuso de substâncias ${ }^{11,52,54,55}$, transtornos mentais orgânicos ${ }^{11}$, ansiedade ${ }^{12}$, autismo ${ }^{55}$

Ansiedade ${ }^{12}$, autismo ${ }^{55}$

\section{Atitudes do paciente}

Não cumpre a medicação ${ }^{11}$

Sinais de tensão, expressões faciais irritadas ${ }^{54}$, humor lábil ${ }^{54}$, irritabilidade ${ }^{54}$, impulsividade ${ }^{53,54}$, hostilidade ${ }^{53,54}$, improdutivo, atividade verbal repetitiva ${ }^{54}$, comportamento não cooperativo ou exigente, resistência ao cuidado ${ }^{54}$, comportamento intimidador ou invasivo ${ }^{54}$, inquietação, torção das mãos, puxar roupas ${ }^{54}$, aumento do volume de fala ou explosões vocai ${ }^{54}$, contato visual prolongado ${ }^{54}$, descontentamento, recusa em se comunicar ${ }^{54}$

Agressor e vítima do mesmo sexo 53

\section{Atitudes do pessoal}

Bloqueio de rotas de fuga ${ }^{54}$, negação por parte da equipe de um pedido ou privilégio do paciente ${ }^{54}$, reforços das regras por equipe/definição de limites $^{54}$, demandas de outros pacientes e funcionários para interromper uma atividade ou concluir uma tarefa ${ }^{54}$ 

SANTOS ${ }^{1,18,19}$, VICENTE DE P. GOMES JÚNIOR ${ }^{1,20}$, QUIRINO CORDEIRO ${ }^{21}$, ANTÔNIO GERALDO DA SILVA ${ }^{22,23,24}$, TENG C. TUNG ${ }^{1,25}$, ALEXANDRE PAIM DIAZZ ${ }^{26,27}$

Tabela 3 - Possíveis testes adicionais que devem estar disponíveis no atendimento a pacientes agitados* $(D)^{56}$

Teste
Albumina
Amilase, gamaglutamil transferase e lipase
Aminotransferase (AST/ALT)
Urinálise (tipo I ou elementos e sedimentos
anormais)
Bilirrubina
Creatinofosfoquinase (CPK)

Amostra

Sangue

Sangue

Sangue

Urina

Sangue

Sangue

Dosagem sérica de medicamento

Eletrocardiograma

Eletrólitos

Análise do líquido cefalorraquidiano

Testes toxicológicos

Sangue

Urina

Saliva

Cabelo

Função renal (ureia e creatinina)

Sangue

Função da tireoide

Sangue

Glicose

Sangue

Urina

Hemograma completo

Imagem cerebral (TC ou RM do encéfalo)

Imagem

Raio X do tórax

Imagem

Sorologias

Sangue

Tempo de protrombina

Sangue

Teste de gravidez

Vitamina B12 e folato

Urina

Sangue

\section{Útil para o diagnóstico de}

Estado nutricional

Distúrbios hepáticos

Doença pancreática

Doença do trato biliar

Ingestão aguda de álcool

Doença hepática

Infecções

Distúrbios metabólicos

Cálculos urinários (cálcio?)

Distúrbios hepáticos

Lesão no músculo esquelético

Síndrome neuroléptica maligna

Dano no músculo cardíaco (infarto)

Acidente vascular encefálico

Avaliação de níveis tóxicos, ou seja, carbamazepina, valproato e lítio

Arritmias, infarto

Alterações nos níveis de eletrólitos, especialmente como causa de delirium

Infecções do sistema nervoso

Neoplasia do sistema nervoso

Hipertensão intracraniana

Doenças desmielinizantes

Hemorragia subaracnóidea

Confirmação de abuso de substâncias

Insuficiência renal devido a infecções

Cálculos renais, envenenamento (lítio), ou outras doenças

Hipo e hipertireoidismo

Hiper ou hipoglicemia

Diagnóstico diferencial de delirium

Anemia, leucocitose ou leucopenia, trombocitopenia e trombocitose

Diagnóstico diferencial de delirium e outras condições orgânicas Transtorno mental - neoplasia cerebral - acidente vascular cerebral Infecções do SNC

Doença pulmonar

Doença cardíaca

Doenças infecciosas

Distúrbios da coagulação

Distúrbios hepáticos

Sangue Gravidez

Anemia megaloblástica

TC = tomografia computadorizada; RM = ressonância magnética; $\mathrm{SNC}$ = sistema nervoso central.

* Os testes devem ser solicitados com base na avaliação clínica. 
uma adequada avaliação física. Assim, os profissionais devem ser orientados pela história do paciente e sinais e sintomas específicos apresentados. O exame deve incluir pelo menos (D): informações dos sinais vitais (temperatura, frequência cardíaca, pressão arterial, frequência respiratória, saturação de oxigênio), sistema cardiovascular, sistema respiratório, sistema gastrointestinal e sistema neurológico ${ }^{57}$. Um exame físico completo pode não ser possível (por exemplo, se o paciente não colaborar, se estiver confuso, violento ou sedado). No entanto, uma observação rápida é sempre possível e pode gerar informações valiosas.

A avaliação da aparência do paciente deve se concentrar nos seguintes aspectos: descrição (alto, baixo, magro, obeso, sexo, idade), odor (álcool, cetona, forte odor corporal), apresentação (arrumado, desgrenhado, barbeado), cicatrizes de automutilação prévia (lembrese de que essas cicatrizes podem estar em locais pouco visíveis, como coxas, abdômen, mamas), abuso de substâncias (marcas), pulseira de alerta médico (epilepsia, diabetes, entre outras enfermidades), sinais óbvios de lesão, atitude (hostil, amigável, agitado, calmo) e coloração (cianótico, pálido). A avaliação neurológica mínima deve incluir avaliação dos movimentos e força dos membros, assimetria facial, tremor, orientação temporoespacial (o paciente está ciente de que está no hospital), nível de consciência (estável ou flutuando), pupilas (forma, tamanho e reatividade) e sinais de traumatismo craniano (recente ou anterior).

$\mathrm{Na}$ avaliação do estado mental/exame psiquiátrico, não existe uma metodologia única para essa observação. Contudo,osprincipais elementos deumexamepsiquiátrico mínimo devem ser realizados: apresentação, atitude, contato, consciência, atenção, orientação, memória, sensopercepção, pensamentos, crítica (consciência de morbidade), humor/afeto e psicomotricidade (D) ${ }^{58}$.

O objetivo aqui não é discutir cada um desses itens. Mais detalhes estão disponíveis na literatura ${ }^{5,17,21,23,24,26,29,58-60}$. Entretanto, as funções psiquiátricas que apresentam maiores alterações devem ser descritas com mais detalhes.

\section{DiAGNÓSTICO DIFERENCIAL}

Oraciocínio clínicoadequadoé essencial parase levantar diagnósticos diferenciais. Muitas manifestações, como agitação psicomotora e agressividade, são inespecíficas e podem estar associadas a diversas condições médicas. Alguns dados da história podem reforçar a hipótese de condições clínicas ou neurológicas (transtorno mental orgânico ou delirium). Os principais são: início abrupto, idade superior a 40 anos, ausência de transtornos psiquiátricos prévios, alucinações visuais, olfativas ou táteis, fala distorcida, confusão mental, desorientação temporoespacial e/ou sinais físicos de trauma ${ }^{59}$.

$\mathrm{Na}$ sala de emergência, pacientes com delirium podem ser erroneamente diagnosticados como sendo psicóticos. Os sinais de delirium, que incluem alteração de nível de consciência e dificuldades atencionais, podem ser sutis e requerem muita atenção para que sejam detectados ${ }^{28}$. As principais condições médicas associadas à agitação psicomotora aguda são: hipoglicemia, hipóxia, trauma encefálico, hemorragia, hipertermia e hipotermia, meningite, sepse, acidente vascular encefálico, hemorragia subaracnóidea, estado epilético, tumores cerebrais, tireoidopatia, entre outras enfermidades ${ }^{59}$. As condições médicas e psiquiátricas que podem causar agitação estão listadas no Quadro 2.

O diagnóstico definitivo não é necessário na avaliação inicial desses pacientes. Porém, o diagnóstico sindrômico e o diagnóstico diferencial são fundamentais para iniciar o tratamento. As principais categorias diagnósticas são: síndrome catatônica, síndrome maniforme, transtorno depressivo, transtorno psicótico, transtornos ansiosos, uso de substâncias e síndrome demencial ${ }^{5}$. O uso abusivo ou a abstinência de substâncias psicoativas podem cursar com alterações comportamentais, como agitação, agressividade e quadros psicóticos. Pacientes com histórico de transtorno psiquiátrico grave, como esquizofrenia ou transtorno afetivo bipolar, costumam fazer uso de serviços de emergências psiquiátricas nas crises. Os episódios maniformes ou psicóticos são caracterizados por alterações do conteúdo do pensamento e da percepção da realidade. Períodos de agitação psicomotora e/ou agressividade também podem ocorrer. Os episódios depressivos podem estar associados a comportamentos agitados e agressivos, além de risco de suicídio. Pacientes com transtornos de personalidade também podem estar em serviços de emergência psiquiátrica, com quadro de agitação ou agressividade por baixa tolerância a frustração e 
impulsividade ${ }^{18}$. As avaliações psicométricas podem ser úteis, como um registro objetivo do diagnóstico diferencial. As escalas devem ser selecionadas caso a caso e não serão discutidas nestas diretrizes.

\section{TESTES COMPLEMENTARES}

Os exames complementares são úteis para se descartar causas orgânicas. Embora não existam exames laboratoriais para confirmar ou descartar condições psiquiátricas, os testes podem ser e são amplamente utilizados para descartar patologias orgânicas com

Quadro 2 - Condições médicas e psiquiátricas que podem causar agitação

\section{Agitação de causa médica geral}

Trauma cranioencefálico

Encefalite, meningite ou outra infecção

Encefalopatia (particularmente de insuficiência hepática ou renal)

Exposição a toxinas ambientais

Distúrbio metabólico (por exemplo, hiponatremia,

hipocalcemia, hipoglicemia)

Hipóxia

Doença da tireoide

Período pós convulsão (pós-ictal)

Níveis tóxicos de medicamentos (por exemplo,

psiquiátricos ou anticonvulsivantes)

\section{Agitação por intoxicação/abstinência}

Álcool

Outras drogas

\section{Agitação por transtorno psiquiátrico}

Transtorno psicótico

Estados maníacos e mistos

Depressão agitada

Transtorno de ansiedade

Transtorno da personalidade

Agitação reativa ou situacional (distúrbio adaptativo)

Transtorno do espectro autista

Agitação indiferenciada (presumidamente de uma condição médica geral até prova em contrário)

Adaptado de Garriga et al. e Nodstrom et al.5,29. manifestações psiquiátricas $5,29,56,57$. Os exames laboratoriais de rotina não são indicados; ao contrário, o teste direcionado deve se basear nos diagnósticos mais prováveis ${ }^{29}$. O tratamento da agitação a partir de uma condição médica geral deve ser direcionado à identificação das causas mais prováveis. A Tabela 3 descreve os exames mais comuns (D) 5,29,56,57.

Os exames de neuroimagem devem ser solicitados sempre que houver suspeita de doença neurológica e em pacientes que apresentem um primeiro episódio psicótico (D). Cuidados extra devem ser tomados se o paciente fizer parte de uma população especial - mulheres grávidas, pessoas em situação de rua, adolescentes, crianças e idosos. Nessas populações, deve-se ter cautela ao avaliar possíveis causas orgânicas (D).

\section{ConCLUSÃo}

Apesar das poucas evidências na literatura sobre a abordagem não farmacológica da agitação psicomotora, é possível sistematizar as informações atualmente disponíveis. É importante que os profissionais da saúde sejam treinados em abordagens não farmacológicas, como parte dos requisitos de graduação e pós-graduação. A possibilidade de transtorno mental orgânico como causa primária ou secundária da alteração comportamental deve ser considerada em primeiro lugar. Deve ser dada atenção especial à aparência e ao comportamento do paciente, sinais físicos e estado mental.

Correspondência: Leonardo Baldaçara, Quadra 401 Sul, Av. Joaquim Teotônio Segurado, 1, Espaço Médico, sala 1006, CEP 77015-550, Plano Diretor Sul, Palmas, TO. Tel.: (63) 3217.7288. E-mail: leonardobaldassara@gmail.com

\section{Referências}

1. Cordeiro DC, Baldacara L. Emergências psiquiátricas. São Paulo: Roca; 2007.

2. Barros RE, Marques JM, Carlotti IP, Zuardi AW, Del-Ben CM. Short admission in an emergency psychiatry unit can prevent prolonged lengths of stay in a psychiatric institution. Braz J Psychiatry. 2010;32:145-51.

3. Cruz CM, Leiva JL, Borgoño RV, Larach TK, Errázuriz LB, Chianale IC, et al. Epidemiología de la Urgencia Psiquiátrica en el Instituto Psiquiátrico 
"Dr. José Horwitz": Un nuevo estudio comparativo. Rev Chil Neuro-psiquiatr. 2010;48:175-83.

4. QuevedoJ,CarvalhoAF.Emergências psiquiátricas. $2^{a}$ ed. Porto Alegre: Artmed; 2014.

5. Garriga M, Pacchiarotti I, Kasper S, Zeller SL, Allen $\mathrm{MH}$, Vázquez $\mathrm{G}$, et al. Assessment and management of agitation in psychiatry: expert consensus. World J Biol Psychiatry. 2016;17:86128.

6. Pascual JC, Madre M, Puigdemont D, Oller S, Corripio I, Díaz A, et al. [A naturalistic study: 100 consecutive episodes of acute agitation in a psychiatric emergency department]. Actas Esp Psiquiatr. 2006;34:239-44.

7. Boudreaux ED, Allen MH, Claassen C, Currier GW, Bertman L, Glick R, et al. The psychiatric emergency research collaboration-01: methods and results. Gen Hosp Psychiatry. 2009;31:51522.

8. Pajonk FG, Schmitt P, Biedler A, Richter JC, Meyer W, Luiz T, et al. Psychiatric emergencies in prehospital emergency medical systems: a prospective comparison of two urban settings. Gen Hosp Psychiatry. 2008;30:360-6.

9. San L, Marksteiner J, Zwanzger P, Figuero MA, Romero FT, Kyropoulos G, et al. State of acute agitation at psychiatric emergencies in Europe: the STAGE study. Clin Pract Epidemiol Ment Health. 2016;12:75-86.

10. Brakoulias V, Mandali R, Seymour J, Sammut $P$, Starcevic $V$. Characteristics of admissions to a recently opened psychiatric emergency care centre. Australas Psychiatry. 2010;18:326-9.

11. Chaput Y, Beaulieu L, Paradis M, Labonte E. Aggressive behaviors in the psychiatric emergency service. Open Access Emerg Med. 2011;3:13-20.

12. Grudnikoff E, Taneli T, Correll CU. Characteristics and disposition of youth referred from schools for emergency psychiatric evaluation. Eur Child Adolesc Psychiatry. 2015;24:731-43.

13. Santos ME, do Amor JA, Del-Ben CM, Zuardi AW. [Psychiatric emergency service in a university general hospital: a prospective study]. Rev Saude Publica. 2000;34:468-74.
14. Padilha VM, Schettini CS, Santos Junior A, Azevedo RC. Profile of patients attended as psychiatric emergencies at a university general hospital. Sao Paulo Med J. 2013;131:398-404.

15. Holloman GH Jr, Zeller SL. Overview of project BETA: best practices in evaluation and treatment of agitation. West J Emerg Med. 2012:13:1-2.

16. Lukens TW, Wolf SJ, Edlow JA, Shahabuddin $\mathrm{S}$, Allen $\mathrm{MH}$, Currier GW, et al. Clinical policy: critical issues in the diagnosis and management of the adult psychiatric patient in the emergency department. Ann Emerg Med. 2006;47:79-99.

17. National Institute for Health and Clinical Excellence (NICE). Violence: the short-term management of disturbed/violent behaviour in psychiatric in- patient settings and emergency departments [Internet]. 2005 Feb [cited 2020 Oct 28]. umh1946.edu.umh.es/wp-content/ uploads/sites/172/2015/04/NICE-Violence-Theshort-term-management-of-disturbed-violentbehaviour-in-in-patient-psychiatric-settings-andemergency-departments.pdf

18. Mantovani C, Migon MN, Alheira FV, Del-Ben CM. [Management of the violent or agitated patient]. Braz J Psychiatry. 2010;32 Suppl 2:S96-103.

19. Schleifer JJ. Management of acute agitation in psychosis: an evidence-based approach in the USA. Adv Psychiatr Treat. 2011;17:91-100.

20. Kawakami D, Prates JG, Tung TC. Propostas para o futuro: estrutura física e equipe ideal nas emergências psiquiátricas. Debates Psiquiatr. 2016;6:28-34.

21. Mental Health Division, WA Department of Health. Guidelines: the management of disturbed/ violent behaviour in inpatient psychiatric settings. Perth: Department of Health; 2006.

22. Richmond JS, Berlin JS, Fishkind AB, Holloman GH Jr, Zeller SL, Wilson MP, et al. Verbal de-escalation of the agitated patient: consensus statement of the american association for emergency psychiatry project BETA de-escalation workgroup. West J Emerg Med. 2012;13:17-25.

23. Marder SR. A review of agitation in mental illness: treatment guidelines and current therapies. J Clin Psychiatry. 2006;67 Suppl 10:13-21. 
24. Caldieraro MA, Spode A, Fleck MPA. Avaliação do paciente na emergência. In: Quevedo J, Schimitt R, Kapczinski F. Emergências psiquiátricas. Porto Alegre: Artmed; 2008. p. 7-48.

25. O'Brien L, Cole R. Close-observation areas in acute psychiatric units: a literature review. Int J Ment Health Nurs. 2003;12:165-76.

26. Baldaçara L, Cordeiro DC, Calfat ELB, Cordeiro DC, Chung TC. Emergências psiquiátricas. Rio de Janeiro: Elsevier; 2019.

27. Baldacara L, Ismael F, Leite V, Pereira LA, Dos Santos RM, Gomes VP Jr, et al. Brazilian guidelines for the management of psychomotor agitation. Part 1. Non-pharmacological approach. Braz J Psychiatry. 2019;41:153-67.

28. Stowell KR, Florence P, Harman HJ, Glick RL. Psychiatric evaluation of the agitated patient: consensus statement of the american association for emergency psychiatry project Beta psychiatric evaluation workgroup. West J Emerg Med. 2012;13:11-6.

29. Nordstrom K, Zun LS, Wilson MP, Stiebel V, Ng AT, Bregman B, et al. Medical evaluation and triage of the agitated patient: consensus statement of the american association for emergency psychiatry project Beta medical evaluation workgroup. West J Emerg Med. 2012;13:3-10.

30. Passamar M, Tellier O, Vilamotc B. [Psychomotor agitation, pharmaceutical sedation and psychiatric emergency in psychotic patients]. Encephale. 2011;37:448-56.

31. Strout TD. Psychometric testing of the Agitation Severity Scale for acute presentation behavioral management patients in the emergency department. Adv Emerg Nurs J. 2014;36:250-70.

32. Swift RH, Harrigan EP, Cappelleri JC, Kramer D, Chandler LP. Validation of the behavioural activity rating scale (BARS): a novel measure of activity in agitated patients. J Psychiatr Res. 2002;36:87-95.

33. Almvik R, Woods P. Predicting inpatient violence using the Broset Violence Checklist (BVC). Int J Psychiatr Nurs Res. 1999:4:498-505.

34. Abderhalden C, Needham I, Dassen T, Halfens R, Haug HJ, Fischer J. Predicting inpatient violence using an extended version of the Broset-ViolenceChecklist: instrument development and clinical application. BMC Psychiatry. 2006;6:17.

35. Marques I, Bessa A, Santos L, Carvalho S. Tradução e adaptação da BrØset Violence Checklist [dissertação]. Coimbra; 2004.

36. Huber CG, Lambert M, Naber D, Schacht A, Hundemer HP, Wagner TT, et al. Validation of a Clinical Global Impression Scale for Aggression (CGI-A) in a sample of 558 psychiatric patients. Schizophr Res. 2008;100:342-8.

37. Douglas KS, Ogloff JR, Nicholls TL, Grant I. Assessing risk for violence among psychiatric patients: the HCR-20 violence risk assessment scheme and the Psychopathy Checklist: Screening Version. J Consult Clin Psychol. 1999;67:917-30.

38. Telles LE, Day VP, Folino JO, Taborda JG. Reliability of the Brazilian version of HCR-20 assessing risk for violence. Braz J Psychiatry. 2009;31:253-6.

39. Yudofsky SC, Silver JM, Jackson W, Endicott J, Williams D. The Overt Aggression Scale for the objective rating of verbal and physical aggression. Am J Psychiatry. 1986;143:35-9.

40. Yudofsky SC, Kopecky HJ, Kunik M, Silver JM, Endicott J. The Overt Agitation Severity Scale for the objective rating of agitation. J Neuropsychiatry Clin Neurosci. 1997:9:541-8.

41. Montoya A, Valladares A, Lizan L, San L, Escobar R, Paz S. Validation of the Excited Component of the Positive and Negative Syndrome Scale (PANSSEC) in a naturalistic sample of 278 patients with acute psychosis and agitation in a psychiatric emergency room. Health Qual Life Outcomes. 2011;9:18

42. Sessler CN, Gosnell MS, Grap MJ, Brophy GM, O'Neal PO, Keane KA, et al. The Richmond Agitation-Sedation Scale: validity and reliability in adult intensive care unit patients. Am J Respir Crit Care Med. 2002;166:1338-44.

43. Nassar Junior AP, Pires Neto RC, de Figueiredo WB, Marcelo P. Validity, reliability and applicability of Portuguese versions of sedation-agitation scales among critically ill patients. Sao Paulo Med J. 2008;126:215-9. 
44. Ryder-Lewis MC, Nelson KM. Reliability of the Sedation-Agitation Scale between nurses and doctors. Intensive Crit Care Nurs. 2008;24:211-7.

45. Calver LA, Stokes B, Isbister GK. Sedation assessment tool to score acute behavioural disturbance in the emergency department. Emerg Med Australas. 2011;23:732-40.

46. Hvidhjelm J, Sestoft D, Bjorner JB. The Aggression Observation Short Form identified episodes not reported on the Staff Observation Aggression Scale--Revised. Issues Ment Health Nurs. 2014;35:464-9.

47. Nijman HL, Muris P, Merckelbach HL, Palmstierna T, Wistedt B, Vos AM, et al. The Staff Observation Aggression Scale-Revised (SOAS-R). Aggr Behav. 1999:25:197-209.

48. McNiel DE, Gregory AL, Lam JN, Binder RL, Sullivan GR. Utility of decision support tools for assessing acute risk of violence. J Consult Clin Psychol. 2003;71:945-53.

49. Citrome L, Volavka J. The psychopharmacology of violence: making sensible decisions. CNS Spectr. 2014;19:411-8.

50. Duxbury J, Whittington R. Causes and management of patient aggression and violence: staff and patient perspectives. J Adv Nurs. 2005;50:469-78.

51. Buchanan A, Leese M. Detention of people with dangerous severe personality disorders: a systematic review. Lancet. 2001;358:1955-9.

52. Dack C, Ross J, Papadopoulos C, Stewart D, Bowers $L$. A review and meta-analysis of the patient factors associated with psychiatric in-patient aggression. Acta Psychiatr Scand. 2013;127:255-68.
53. Cornaggia CM, Beghi M, Pavone F, Barale F. Aggression in psychiatry wards: a systematic review. Psychiatry Res. 2011;189:10-20.

54. Hankin CS, Bronstone A, Koran LM. Agitation in the inpatient psychiatric setting: a review of clinical presentation, burden, and treatment. J Psychiatr Pract. 2011;17:170-85.

55. Langstrom N, Grann M, Ruchkin V, Sjostedt G, Fazel S. Risk factors for violent offending in autism spectrum disorder: a national study of hospitalized individuals. J Interpers Violence. 2009;24:135870.

56. Xavier RM, Dora JM, Souza CFM, Barros E. Laboratório na prática clínica consulta rápida. $2^{a}$ ed. Porto Alegre: Artmed; 2011.

57. Mental Health and Drug and Alcohol Office. Mental health for emergency departments a reference guide. Sydney: New South Wales Ministry of Health; 2009.

58. Sanches M, Marques AP, Ortegosa S, Freirias A, Uchida R, Tamai S. O exame do estado mental. É possível sistematizá-lo? Arq Med Hosp Fac Cienc Med Santa Casa São Paulo. 2005:50:18-23.

59. Mantovani C, Labate CM, Sponholz A Jr, Marques JM, Guapo VG, dos Santos ME, et al. Are low doses of antipsychotics effective in the management of psychomotor agitation? A randomized, ratedblind trial of 4 intramuscular interventions. J Clin Psychopharmacol. 2013;33:306-12.

60. Baldacara L, Diaz AP, Leite V, Pereira LA, Dos Santos RM, Gomes Júnior VdP, et al. Brazilian guidelines for the management of psychomotor agitation. Part 2. Pharmacological approach. Braz J Psychiatry. 2019;41:324-35. 